\title{
Deformations of Transverse Calabi-Yau Structures on Foliated Manifolds
}

\author{
by \\ Takayuki MORIYAMA
}

\begin{abstract}
We develop a deformation theory of transverse structures given by calibrations on foliated manifolds, including transverse Calabi-Yau, hyperkähler, $G_{2}$ and $\operatorname{Spin}(7)$ structures. We show that the deformation space of the transverse structures is smooth under a cohomological assumption. As an application, we obtain unobstructed deformations of transverse Calabi-Yau structures on foliated manifolds. We also prove a Moser type stability result for transverse structures, which implies Moser's stability theorem for presymplectic forms.
\end{abstract}

2010 Mathematics Subject Classification: Primary 58H15; Secondary 53C12.

Keywords: foliation, transverse Calabi-Yau structure, deformation theory.

\section{$\S 1$. Introduction}

Kodaira and Spencer worked out the deformation theory of compact complex manifolds [10. They showed that there exists a family of deformations of complex structures parameterized by a smooth finite-dimensional space which is versal, under a cohomological assumption. Kuranishi proved a general theorem on the existence of a versal deformation space for a given complex structure, where the versal deformation space (Kuranishi space) is given by an analytic space which is not necessarily smooth [11. Duchamp-Kalka 4] and Gomez-Mont 7] showed a weak version of Kuranishi's theorem for deformations of transversely holomorphic foliations on compact manifolds. Girbau, Haefliger and Sundararaman 6] constructed the Kuranishi space of deformations of transversely holomorphic foliations on compact manifolds.

In this paper, we introduce a different deformation theory of transverse geometric structures on foliated manifolds. We consider transverse structures defined in terms of systems of closed forms called transverse calibrations (see Def-

Communicated by H. Nakajima. Received December 17, 2008. Revised September 1, 2009.

T. Moriyama: RIMS, Kyoto University, Kyoto 606-8502, Japan;

e-mail: moriyama@kurims.kyoto-u.ac.jp

(C) 2010 Research Institute for Mathematical Sciences, Kyoto University. All rights reserved. 
inition 1.1). In the theory of Riemannian holonomy groups, it is useful to characterize geometric structures as systems of closed forms. From this point of view, McLean [12] produced the deformation theory of compact special Lagrangian submanifolds. Goto [12] discussed a deformation space of calibrations defined by closed differential forms. By considering Calabi-Yau, hyperkähler, $G_{2}$ and $\operatorname{Spin}(7)$ structures as calibrations, he showed that these deformations are unobstructed. We fix a foliation on a manifold and deform the transverse calibrations on it. One of the advantages of our approach is that we can use Hodge theory on a foliated manifold [5]. (Recently, this transverse version of Hodge theory plays an important role in Sasakian geometry [2].) As a result, in a suitable case such as transverse Calabi-Yau, hyperkähler, $G_{2}$ and $\operatorname{Spin}(7)$ structures, we obtain a smooth deformation space of the structures. We can also generalize Moser's theorem in symplectic geometry to transverse calibrations, and prove Moser's stability theorem for presymplectic forms. (We refer to [3] for another generalization of Moser's theorem given by Crainic and Fernandes in Poisson geometry.)

Let $M$ be a compact manifold of dimension $p+q$ and $\mathcal{F}$ a foliation on $M$ of codimension $q$. The foliation $\mathcal{F}$ is defined by data $\left\{U_{i}, f_{i}, T, \gamma_{i j}\right\}$ consisting of an open covering $\left\{U_{i}\right\}_{i}$ of $M$, a $q$-dimensional transverse manifold $T$, submersions $f_{i}: U_{i} \rightarrow T$ and diffeomorphisms $\gamma_{i j}: f_{i}\left(U_{i} \cap U_{j}\right) \rightarrow f_{j}\left(U_{i} \cap U_{j}\right)$ for $U_{i} \cap U_{j} \neq \emptyset$ satisfying $f_{j}=\gamma_{i j} \circ f_{i}$. A transverse structure on $(M, \mathcal{F})$ is a geometric structure on $T$ which is invariant under $\gamma_{i j}$. For example, a transverse Kähler structure is defined by a Kähler structure on $T$ preserved by $\gamma_{i j}$. A foliation $\mathcal{F}$ is called transverse Kähler if there exists a transverse Kähler structure on $(M, \mathcal{F})$. On the compact manifold $M$ with a transverse Kähler foliation $\mathcal{F}$, if the basic canonical line bundle is trivial, then there exists a transverse Calabi-Yau structure on $(M, \mathcal{F})$ by applying the basic version of Yau's theorem [5]. Alternative definitions for such transverse structures can be given in terms of basic sections of basic bundles over $(M, \mathcal{F})$ (see Section 2). In particular, any transverse Calabi-Yau structure is characterized by a pair of closed basic forms (see Definition 6.3).

We discuss the deformation space of transverse Calabi-Yau structures on $(M, \mathcal{F})$ by introducing Goto's deformation theory of calibrations to the geometry of foliations. Our idea is to consider basic differential forms on $(M, \mathcal{F})$ instead of differential forms on $M$. Let $W$ be a $q$-dimensional vector space and $\bigwedge^{p} W^{*}$ the space of skew-symmetric tensors of the dual space $W^{*}$. Then the group $G=\operatorname{GL}(W)$ acts diagonally on the direct sum $\bigoplus_{i=1}^{\ell} \bigwedge^{p_{i}} W^{*}$. Let $\Phi_{W}=\left(\phi_{1}, \ldots, \phi_{\ell}\right)$ be an element of $\bigoplus_{i=1}^{\ell} \bigwedge^{p_{i}} W^{*}$ and $\mathcal{O}\left(=\mathcal{A}_{\mathcal{O}}(W)\right)$ the $G$-orbit through $\Phi_{W}$ with an isotropy group $H$, so $\mathcal{O}$ is the homogeneous space $G / H$. On the foliated manifold $(M, \mathcal{F})$, we have a completely integrable distribution $F$ of dimension $p$ and the quotient bundle $Q=T M / F$ over $M$. Let $\mathcal{A}_{\mathcal{O}}(M, \mathcal{F})$ be the fiber bundle $\bigcup_{x \in M} \mathcal{A}_{\mathcal{O}}\left(Q_{x}\right)$ 
and $\mathcal{E}_{\mathcal{O}}$ the set $\Gamma\left(M, \mathcal{A}_{\mathcal{O}}(M, \mathcal{F})\right) \cap \bigoplus_{i=1}^{\ell} \bigwedge_{\mathrm{B}}^{p_{i}}$ of sections of $\mathcal{A}_{\mathcal{O}}(M, \mathcal{F})$ defined to be basic forms, where $\bigwedge_{\mathrm{B}}^{p_{i}}$ denotes the space of basic $p_{i}$-forms on $M$.

Definition 1.1. A system $\Phi$ of differential forms on $(M, \mathcal{F})$ is called a transverse calibration associated with the orbit $\mathcal{O}$ if $\Phi$ is an element of $\mathcal{E}_{\mathcal{O}}$ whose components are closed as differential forms.

Let $\widetilde{\mathfrak{M}}_{\mathcal{O}}(M, \mathcal{F})$ be the set of transverse calibrations associated with $\mathcal{O}$. We can identify $\widetilde{\mathfrak{M}}_{\mathcal{O}}(M, \mathcal{F})$ with the intersection $\mathcal{E}_{\mathcal{O}} \cap \mathcal{H}$ where $\mathcal{H}$ is the Hilbert space consisting of all closed forms on $M$. If we consider $\mathcal{E}_{\mathcal{O}}$ as a Hilbert manifold, then the infinitesimal tangent space of $\widetilde{\mathfrak{M}}_{\mathcal{O}}(M, \mathcal{F})$ at $\Phi$ is regarded as the intersection $T_{\Phi} \mathcal{E}_{\mathcal{O}} \cap \mathcal{H}$. We investigate whether the infinitesimal tangent space is identified with the tangent space of actual deformations.

Definition 1.2. A transverse calibration $\Phi \in \widetilde{\mathfrak{M}}_{\mathcal{O}}(M, \mathcal{F})$ is unobstructed if for any $\alpha \in T_{\Phi} \mathcal{E}_{\mathcal{O}} \cap \mathcal{H}$, there exists an integral curve $\Phi_{t}$ in $\widetilde{\mathfrak{M}}_{\mathcal{O}}(M, \mathcal{F})$ such that

$$
\left.\frac{d}{d t} \Phi_{t}\right|_{t=0}=\alpha
$$

For $\Phi \in \widetilde{\mathfrak{M}}_{\mathcal{O}}(M, \mathcal{F})$, we define in Section 3.2 a complex $\left(\sharp_{\Phi}\right)$ and maps

$$
p^{k}: H^{k}\left(\sharp_{\Phi}\right) \rightarrow \bigoplus_{i} H_{\mathrm{B}}^{p_{i}+k-1}(M)
$$

for each $k \geq 0$, where $H_{\mathrm{B}}^{k}(M)$ is the basic de Rham cohomology group on $(M, \mathcal{F})$. We also define the ellipticity of an orbit $\mathcal{O}$ (Definition 3.1). Then we obtain the main theorem:

Theorem 1.1. Let $\mathcal{O}$ be an elliptic orbit. If $\Phi \in \widetilde{\mathfrak{M}}_{\mathcal{O}}(M, \mathcal{F})$ and the map $p^{2}$ : $H^{2}\left(\sharp_{\Phi}\right) \rightarrow \bigoplus_{i} H_{\mathrm{B}}^{p_{i}+1}(M)$ is injective, then $\Phi$ is unobstructed.

We can regard a transverse Calabi-Yau structure on $(M, \mathcal{F})$ as a transverse calibration associated with the orbit $\mathcal{O}_{\mathrm{CY}}$ of Calabi-Yau structures. Then we obtain

Theorem 1.2. Any transverse Calabi-Yau structure on $(M, \mathcal{F})$ is unobstructed.

In Section 2, we prepare some results in foliated geometry and provide definitions of "basic" objects, for example, basic vector bundles, basic sections, etc. In Section 3, we introduce transverse calibrations on $(M, \mathcal{F})$. Each transverse calibration induces a deformation complex. Then we see that the deformation complex is a subcomplex of the basic de Rham complex. In Section 4, we provide a sufficient condition for the transverse structures to have unobstructed deformations (Theorem 4.1. We consider a generalization of Moser's stability theorem (Theorem 5.1) in Section 5. As an application, we obtain Moser's stability theorem for presym- 
plectic forms and prove that the deformations of transverse Calabi-Yau structures on $(M, \mathcal{F})$ are unobstructed in the last section.

\section{§2. Preparations on foliated geometry}

In this section, we provide some results in foliated geometry. For these results and the notation, we refer to [1], 14] and [13. We assume that $M$ is a compact manifold of dimension $p+q$ and $\mathcal{F}$ is a foliation on $M$ of codimension $q$. We denote by $F$ a completely integrable distribution of dimension $p$ associated to the foliation $\mathcal{F}$. Let $Q$ be the normal bundle $T M / F$ and $\pi: T M \rightarrow Q$ the natural projection.

\section{$\S 2.1$. Basic vector fields and basic forms}

A vector field $u \in \Gamma(T M)$ is foliated if $[u, v] \in \Gamma(F)$ for any $v \in \Gamma(F)$. We denote by $\Gamma(M, \mathcal{F})$ the set of foliated vector fields on $(M, \mathcal{F})$. Let $\mathfrak{X}(M, \mathcal{F})$ be the quotient space of $\Gamma(M, \mathcal{F})$ by $\Gamma(\mathcal{F})$ :

$$
\mathfrak{X}(M, \mathcal{F})=\Gamma(M, \mathcal{F}) / \Gamma(F)
$$

We call an element $u$ of $\mathfrak{X}(M, \mathcal{F})$ a basic vector field on $(M, \mathcal{F})$.

A differential $k$-form $\phi \in \bigwedge^{k}$ on $M$ is a basic form on $(M, \mathcal{F})$ if the interior product $i_{v} \phi$ and the Lie derivative $L_{v} \phi$ vanish for any $v \in \Gamma(F)$. Let $\bigwedge_{\mathrm{B}}^{k}$ be the set of basic $k$-forms on $(M, \mathcal{F})$ :

$$
\bigwedge_{\mathrm{B}}^{k}=\left\{\phi \in \bigwedge^{k} \mid i_{v} \phi=L_{v} \phi=0, \forall v \in \Gamma(F)\right\}
$$

For a section $u \in \Gamma(Q)$ and a basic $k$-form $\phi \in \bigwedge_{\mathrm{B}}^{k}$, the interior product $i_{u} \phi$ and the Lie derivative $L_{u} \phi$ are defined by the $(k-1)$-form $i_{\tilde{u}} \phi$ and the $k$-form $L_{\tilde{u}} \phi$ for a lift $\tilde{u} \in \Gamma(T M)$ of $u$, respectively. If $u$ is a basic vector field, then $i_{u} \phi$ and $L_{u} \phi$ are basic forms.

We define a foliated diffeomorphism to be a diffeomorphism $f$ of $M$ preserving the foliation $\mathcal{F}$, i.e., $f_{*}(F)=F$. We denote by $\operatorname{Diff}(M, \mathcal{F})$ the group of foliated diffeomorphisms:

$$
\operatorname{Diff}(M, \mathcal{F})=\left\{f \in \operatorname{Diff}(M) \mid f_{*}(F)=F\right\} .
$$

We can define an action of $\operatorname{Diff}(M, \mathcal{F})$ on the space of basic forms $\bigwedge_{\mathrm{B}}^{*}$ by pullback. For $u \in \mathfrak{X}(M, \mathcal{F})$, any lift $\tilde{u} \in \Gamma(T M)$ of $u$ induces a one-parameter family of foliated diffeomorphisms $f_{t}$. Then the Lie derivative $L_{u} \phi$ for $\phi \in \bigwedge_{\mathrm{B}}^{k}$ may be regarded as the limit $\left.\frac{d}{d t} f_{t}^{*} \phi\right|_{t=0}$.

\section{$\S 2.2$. Basic bundles and basic sections}

Let $\iota: P \rightarrow M$ be a principal fiber bundle and $\omega$ a connection form on $P$. The horizontal subbundle $H$ is defined by the subbundle $\operatorname{Ker} \omega$ of the tangent bundle $T P$. 
Then the derivative $\iota_{*}$ restricted to $H$ is the isomorphism from $H$ to $T M$. Hence we have the subbundle $\tilde{F}=\iota_{*}^{-1}(F)$ of $H$ over $P$. If $\tilde{F}$ is integrable, then $\tilde{F}$ induces the foliation $\tilde{\mathcal{F}}$ on $P$.

Definition 2.1. A principal fiber bundle $P$ is foliated if there exists a connection form $\omega$ on $P$ such that the bundle $\tilde{F}$ is integrable. Moreover, if the form $\omega$ is basic with respect to the induced foliation $\tilde{\mathcal{F}}$, then the bundle $P$ is called basic.

A vector bundle $\pi: E \rightarrow M$ is called foliated (resp. basic) if the associated principal bundle $P_{E}$ is a foliated (resp. basic) bundle. In the case $\pi: E \rightarrow M$ is a foliated vector bundle, the bundle $P_{E} \rightarrow M$ admits a foliation $\tilde{\mathcal{F}}$ on the total space $P_{E}$ by the definition. This foliation $\tilde{\mathcal{F}}$ induces a foliation $\tilde{\mathcal{F}}_{E}$ on $E$. In addition, if $E$ is basic then there exists a connection $\nabla$ of $E$ whose connection form is basic. Such a connection $\nabla$ is called a basic connection on $E$.

Definition 2.2. Let $E$ be a basic vector bundle with a basic connection $\nabla$. A section $s \in \Gamma(E)$ is called basic if $\nabla_{v} s=0$ for any $v \in \Gamma(F)$.

We denote by $\Gamma_{\mathrm{B}}(E)$ the set of basic sections of $E$. Observe that for a basic bundle $E$, the dual bundle $E^{*}$ and the exterior powers $\bigwedge^{*} E^{*}$ are also basic bundles. We consider a hermitian metric $h$ on $E$ as a section of a basic bundle. Then we call $E$ a basic hermitian bundle if the hermitian metric $h$ is basic.

\section{$\S 2.3$. Riemannian foliations}

We define an action of $\Gamma(F)$ on any section $u \in \Gamma(Q)$ as follows:

$$
L_{v} u=\pi[\tilde{u}, v] \in \Gamma(Q)
$$

for any vector field $v \in \Gamma(F)$ where $\tilde{u} \in \Gamma(T M)$ is a lift of $u$, i.e., a vector field $u \in \Gamma(T M)$ with $\pi(\tilde{u})=u$. This action is independent of the choice of lifts $\tilde{u} \in \Gamma(T M)$ of $u$. Let $g$ be a Riemannian metric on $M$. Then we have an orthogonal decomposition $T M=F^{\perp} \oplus_{g} F$ and the isomorphism $\sigma: Q \rightarrow F^{\perp}$. We denote by $S^{2} Q^{*}$ the symmetric 2-tensors of $Q^{*}$. Set a metric $g_{Q}=\sigma^{*} g_{F^{\perp}} \in \Gamma\left(S^{2} Q^{*}\right)$ for the induced metric $g_{F^{\perp}}$ on $F^{\perp}$. Then the map $\sigma:\left(Q, g_{Q}\right) \rightarrow\left(F^{\perp}, g_{F^{\perp}}\right)$ is an isometry. Let $\nabla^{M}$ be the Levi-Civita connection with respect to $g$. Then we introduce a connection $\nabla$ on $Q$ as follows:

$$
\nabla_{v} u= \begin{cases}L_{v} u, & v \in \Gamma(F), \\ \pi\left(\nabla_{v}^{M} \tilde{u}\right), & v \in \Gamma\left(F^{\perp}\right),\end{cases}
$$

for $u \in \Gamma(Q)$, where $\tilde{u} \in \Gamma(T M)$ is a lift of $u$. In general, the connection 2.1 is not necessarily basic. 
A foliation $\mathcal{F}$ is Riemannian if the data $\left\{U_{i}, f_{i}, T, \gamma_{i, j}\right\}$ are such that $T$ is a Riemannian manifold and each $\gamma_{i, j}$ is an isometry. A Riemannian metric $g$ is called bundle-like if $L_{v} g_{Q}=0$ for any $v \in \Gamma(F)$ where the tensor $L_{v} g_{Q} \in \Gamma\left(S^{2} Q^{*}\right)$ is defined by

$$
\left(L_{v} g_{Q}\right)(u, w)=v\left(g_{Q}(u, w)\right)-g_{Q}\left(L_{v} u, w\right)-g_{Q}\left(u, L_{v} w\right)
$$

for $u, w \in \Gamma(Q)$. It turns out that $\mathcal{F}$ is a Riemannian foliation if and only if there exists a bundle-like Riemannian metric $g$ on $M$. For a bundle-like metric $g$, the connection $\nabla$ in $(2.1)$ is basic. Hence $Q$ is a basic vector bundle for a Riemannian foliation $\mathcal{F}$. It is easy to see that any basic section of $\bigwedge^{k} Q^{*}$ is a basic $k$-form on $M$ :

$$
\bigwedge_{\mathrm{B}}^{k}=\Gamma_{\mathrm{B}}\left(\bigwedge^{k} Q^{*}\right)
$$

The space $\Gamma_{\mathrm{B}}(Q)$ is nothing but $\mathfrak{X}(M, \mathcal{F})$ :

$$
\mathfrak{X}(M, \mathcal{F})=\Gamma_{\mathrm{B}}(Q) .
$$

So we also call an element $s$ of $\Gamma_{\mathrm{B}}(Q)$ a basic vector field. Moreover, a basic vector field $s \in \Gamma_{\mathrm{B}}(Q)$ is identified with a foliated vector field $u_{s}=\sigma(s) \in \Gamma\left(F^{\perp}\right)$ by the isomorphism $\sigma$. Therefore we have the following identification:

$$
\Gamma_{\mathrm{B}}(Q) \simeq\left\{u \in \Gamma\left(F^{\perp}\right) \mid[u, v] \in \Gamma(F), \forall v \in \Gamma(F)\right\} .
$$

From now, we consider any basic section of $Q$ as a vector field on $M$ under the identification of $(2.3)$. Then a basic vector field $u \in \Gamma_{\mathrm{B}}(Q)$ induces a one-parameter family of foliated diffeomorphisms $f_{t}$ since a vector field $u \in \Gamma\left(F^{\perp}\right)$ yields a oneparameter family of diffeomorphisms.

\section{§2.4. Transversely elliptic operators}

Let $E$ be a basic bundle of rank $N$. A linear map $D: \Gamma_{\mathrm{B}}(E) \rightarrow \Gamma_{\mathrm{B}}(E)$ is called a basic differential operator of order $\ell$ if, in local coordinates $\left(x_{1}, \ldots, x_{p}, y_{1}, \ldots, y_{q}\right)$ for which $\mathcal{F}$ is given by the equations $d y_{1}=\cdots=d y_{q}=0, D$ has the following expression:

$$
D=\sum_{|s| \leq \ell} a_{s}(y) \frac{\partial^{|s|}}{\partial^{s_{1}} y_{1} \ldots \partial^{s_{q}} y_{q}},
$$

where $s=\left(s_{1}, \ldots, s_{q}\right) \in \mathbb{N}^{q}$ and each $a_{s}$ is an $N \times N$-matrix valued basic function. We define the principal symbol of $D$ at $z=(x, y)$ and the basic covector $\xi \in Q_{z}^{*}$ as the linear map $\sigma(D)(z, \xi): E_{z} \rightarrow E_{z}$ given by

$$
\sigma(D)(z, \xi)(\eta)=\sum_{|s|=\ell} \xi_{1}^{s_{1}} \ldots \xi_{q}^{s_{q}} a_{s}(y)(\eta)
$$

for any $\eta \in E_{z}$. 
Definition 2.3. A basic differential operator $D$ is transversely elliptic if $\sigma(D)(z, \xi)$ is an isomorphism for every $z \in M$ and $\xi(\neq 0) \in Q_{z}^{*}$.

We suppose that $E$ is a hermitian basic bundle with a hermitian metric $h$ and $\ell=2 \ell^{\prime}$. Then a quadratic form $A(D)(z, \xi): E_{z} \rightarrow \mathbb{C}$ is given by

$$
A(D)(z, \xi)(\eta)=(-1)^{\ell^{\prime}}\langle\sigma(D)(z, \xi)(\eta), \eta\rangle \text {. }
$$

Definition 2.4. A basic differential operator $D$ is strongly transversely elliptic if $A(D)(z, \xi)$ is positive definite for every $z \in M$ and every non-zero $\xi \in Q_{z}^{*}$.

Let $\left\{\left(E^{k}, D_{k}\right)\right\}_{k=0,1, \ldots, q}$ be a family of hermitian basic bundles and basic differential operators of order 1 with the differential complex

$$
\cdots \stackrel{D_{k-1}}{\longrightarrow} \Gamma_{\mathrm{B}}\left(E^{k}\right) \stackrel{D_{k}}{\longrightarrow} \Gamma_{\mathrm{B}}\left(E^{k+1}\right) \stackrel{D_{k+1}}{\longrightarrow} \cdots
$$

where $D_{k}: \Gamma_{\mathrm{B}}\left(E^{k}\right) \rightarrow \Gamma_{\mathrm{B}}\left(E^{k}\right)$ for $k=0,1, \ldots, q$. We denote by $\sigma_{k}$ the principal symbol $\sigma\left(D_{k}\right)(z, \xi)$ of $D_{k}$. Then the complex (2.4) is transversely elliptic if the symbol sequence

$$
\cdots \stackrel{\sigma_{k-1}}{\longrightarrow} E_{z}^{k} \stackrel{\sigma_{k}}{\longrightarrow} E_{z}^{k+1} \stackrel{\sigma_{k+1}}{\longrightarrow} \cdots
$$

is exact for any $z$ and any non-zero $\xi$. Note that the complex $(2.4)$ is transversely elliptic if and only if the basic operator $L_{k}=D_{k}^{*} D_{k}+D_{k-1} D_{k-1}^{*}$ is strongly transversely elliptic, where $D_{k}^{*}$ is the formal adjoint operator. We have the Hodge theory for the transversely elliptic complex 2.4 with the cohomology $H_{\mathrm{B}}^{k}\left(E^{*}\right)$ :

Proposition 2.1 ([1, Theorem 15]). (i) The kernel $\mathcal{H}_{\mathrm{B}}^{k}$ of $L_{k}$ is finite-dimensional and we have an orthogonal decomposition

$$
\Gamma_{\mathrm{B}}\left(E^{k}\right)=\mathcal{H}_{\mathrm{B}}^{k} \oplus \operatorname{Im}\left(D_{k-1}\right) \oplus \operatorname{Im}\left(D_{k}^{*}\right) .
$$

(ii) The orthogonal projection $\Gamma_{\mathrm{B}}\left(E^{k}\right) \rightarrow \mathcal{H}_{\mathrm{B}}^{k}$ induces an isomorphism from $H_{\mathrm{B}}^{k}\left(E^{*}\right)$ to $\mathcal{H}_{\mathrm{B}}^{k}$.

\section{$\S 2.5$. Transverse Riemannian structures}

A Riemannian foliation is characterized by the following structure:

Definition 2.5. A symmetric 2-tensor $\tilde{g} \in \Gamma\left(\bigotimes^{2} Q^{*}\right)$ is a transverse Riemannian structure on $(M, \mathcal{F})$ if $\tilde{g}$ is positive definite on $Q$ and satisfies $L_{v} \tilde{g}=0$ for any $v \in \Gamma(F)$ where $L_{v} \tilde{g}$ is defined by 2.2 .

A bundle-like metric $g$ induces a transverse Riemannian structure $g_{Q}$ on $(M, \mathcal{F})$. Conversely, for a transverse Riemannian structure $\tilde{g}$, we can take a bundlelike metric $g$ such that $g_{Q}=\tilde{g}$. Given a transverse Riemannian structure $g_{Q}$ on 
$(M, \mathcal{F})$, the complexification $Q \otimes \mathbb{C}$ is a basic hermitian bundle, and hence so is $\bigwedge^{k} Q^{*} \otimes \mathbb{C}$. Thus from Proposition 2.1 we have

Proposition 2.2 ([1, Theorem 16]). (i) The kernel $\mathcal{H}_{\mathrm{B}}^{k}$ of the basic Laplacian $d d^{*}+d^{*} d$ on $\bigwedge_{\mathrm{B}}^{k}$ is finite-dimensional and we have an orthogonal decomposition

$$
\bigwedge_{\mathrm{B}}^{k}=\mathcal{H}_{\mathrm{B}}^{k} \oplus \operatorname{Im}(d) \oplus \operatorname{Im}\left(d^{*}\right)
$$

(ii) The orthogonal projection $\bigwedge_{\mathrm{B}}^{k} \rightarrow \mathcal{H}_{\mathrm{B}}^{k}$ induces an isomorphism from the basic de Rham cohomology $H_{\mathrm{B}}^{k}(M)$ to $\mathcal{H}_{\mathrm{B}}^{k}$.

\section{§2.6. Transverse Kähler structures}

We can define an action of $\Gamma(F)$ on any section $J \in \Gamma(\operatorname{End}(Q))$ as follows:

$$
\left(L_{v} J\right)(u)=L_{v}(J(u))-J\left(L_{v} u\right)
$$

for $v \in \Gamma(F)$ and $u \in \Gamma(Q)$. If $J \in \Gamma(\operatorname{End}(Q))$ is a complex structure of $Q$, i.e. $J^{2}=-\operatorname{id}_{Q}$, and if $L_{v} J=0$ for any $v \in \Gamma(F)$, then a tensor $N_{J} \in \Gamma\left(\otimes^{2} Q^{*} \otimes Q\right)$ can be defined by

$$
N_{J}(u, w)=[J u, J w]_{Q}-[u, w]_{Q}-J[u, J w]_{Q}-J[J u, w]_{Q}
$$

for $u, w \in \Gamma(Q)$, where $[u, w]_{Q}$ denotes the bracket $\pi[\tilde{u}, \tilde{w}]$ for any lifts $\tilde{u}$ and $\tilde{w}$.

Definition 2.6. A section $J \in \Gamma(\operatorname{End}(Q))$ is a transverse complex structure on $(M, \mathcal{F})$ if $J$ is a complex structure of $Q$, i.e. $J^{2}=-\operatorname{id}_{Q}$, such that $L_{v} J=0$ for any $v \in \Gamma(F)$ and $N_{J}=0$.

A foliation $\mathcal{F}$ is transversely holomorphic if and only if there exists a transverse complex structure on $(M, \mathcal{F})$. Thus we may regard a transverse complex structure as a generalization of complex structures on complex manifolds. A transverse complex structure $J$ on $(M, \mathcal{F})$ give rises to the decomposition $\bigwedge_{\mathrm{B}}^{k} \otimes \mathbb{C}=\bigoplus_{r+s=k} \bigwedge_{\mathrm{B}}^{r, s}$ in the same manner as in complex geometry. We denote by $H_{\mathrm{B}}^{r, s}(M)$ the $(r, s)$ basic Dolbeault cohomology group. We provide the following remark about the integrability condition of a transverse complex structure.

Remark. Let $J$ be a complex structure of $Q$ such that $L_{v} J=0$ for any $v \in \Gamma(F)$. Then $J$ is a transverse complex structure, i.e. $N_{J}=0$, if and only if $d\left(\bigwedge_{\mathrm{B}}^{1,0}\right) \subset$ $\bigwedge_{\mathrm{B}}^{2,0} \oplus \bigwedge_{\mathrm{B}}^{1,1}$, which is equivalent to $d\left(\bigwedge_{\mathrm{B}}^{0,1}\right) \subset \bigwedge_{\mathrm{B}}^{1,1} \oplus \bigwedge_{\mathrm{B}}^{0,2}$, where $d$ denotes the exterior derivative.

Definition 2.7. A pair of sections $(\tilde{g}, J) \in \Gamma\left(\otimes^{2} Q^{*}\right) \times \Gamma(\operatorname{End}(Q))$ is a transverse Kähler structure on $(M, \mathcal{F})$ if $\tilde{g}$ is a transverse Riemannian structure and $J$ is a 
transverse complex structure on $(M, \mathcal{F})$ such that

$$
\tilde{g}(\cdot, J \cdot) \text { is a } d \text {-closed form and } \tilde{g}(J u, J w)=\tilde{g}(u, w)
$$

for $u, w \in \Gamma(Q)$.

A transversely Kähler foliation $\mathcal{F}$ is defined by data $\left\{U_{i}, f_{i}, T, \gamma_{i j}\right\}$ with a Kähler manifold $T$ and local diffeomorphisms $\gamma_{i j}$ preserving the Kähler structure. We remark that there exists a transverse Kähler structure on $(M, \mathcal{F})$ if and only if $\mathcal{F}$ is a transverse Kähler foliation. Given a transverse Kähler structure $(\tilde{g}, J)$, the bundles $\bigwedge_{\mathrm{B}}^{k} \otimes \mathbb{C}$ and $\bigwedge_{\mathrm{B}}^{r, s}$ are all basic hermitian bundles. Then each basic Dolbeault cohomology group $H_{\mathrm{B}}^{r, s}(M)$ is finite-dimensional from Proposition 2.1. Moreover, the basic de Rham-Hodge decomposition holds:

Proposition 2.3 ([1, Theorem 17]). Let $\mathcal{F}$ be a transverse Kähler foliation on $M$. Then there exists an isomorphism

$$
H_{\mathrm{B}}^{k}(M, \mathbb{C})=\bigoplus_{r+s=k} H_{\mathrm{B}}^{r, s}(M) .
$$

\section{$\S 3$. Transverse calibrations}

\section{$\S 3.1$. Orbits in vector spaces}

Let $W$ be a vector space of dimension $q$. We choose $\ell$ natural numbers $\left(p_{1}, \ldots, p_{\ell}\right)$ $\in \mathbb{N}^{\ell}$. We denote by $\rho$ the representation of $G=\mathrm{GL}(\mathrm{W})$ on the space $\bigoplus_{i=1}^{\ell} \bigwedge^{p_{i}} W^{*}$ where each $\bigwedge^{p_{i}} W^{*}$ is the space of skew-symmetric tensors of degree $p_{i}$ of the dual space $W^{*}$. We fix an element $\Phi_{W}=\left(\phi_{1}, \ldots, \phi_{\ell}\right) \in \bigoplus_{i=1}^{\ell} \bigwedge^{p_{i}} W^{*}$ and denote by $H$ the isotropy group of $\Phi_{W}$ :

$$
H=\left\{g \in G \mid \rho_{g} \Phi_{W}=\Phi_{W}\right\}
$$

Then the $G$-orbit

$$
\mathcal{O}=\left\{\rho_{g} \Phi_{W} \in \bigoplus_{i=1}^{\ell} \bigwedge^{p_{i}} W^{*} \mid g \in G\right\}
$$

through $\Phi_{W}$ is regarded as the homogeneous space $G / H$. We denote by $\mathcal{A}_{\mathcal{O}}(W)$ the $G$-orbit $\mathcal{O}$ through $\Phi_{W}$. For an element $\Phi_{0} \in \mathcal{A}_{\mathcal{O}}(W)$, the tangent space $T_{\Phi_{0}} \mathcal{A}_{\mathcal{O}}(W)$ is given by

$$
E_{\Phi_{0}}^{1}(W)=\left\{\hat{\rho}_{\xi} \Phi_{0} \in \bigoplus_{i=1}^{\ell} \bigwedge^{p_{i}} W^{*} \mid \xi \in \mathfrak{g}\right\},
$$

where $\mathfrak{g}$ is the Lie algebra of $G$ and $\hat{\rho}$ is the differential representation of $\mathfrak{g}$. We also define vector spaces $E_{\Phi_{0}}^{0}(W)$ and $E_{\Phi_{0}}^{k}(W)$ by

$$
\begin{aligned}
& E_{\Phi_{0}}^{0}(W)=\left\{i_{v} \Phi_{0}=\left(i_{v} \phi_{1}, \ldots, i_{v} \phi_{\ell}\right) \in \bigoplus_{i=1}^{\ell} \bigwedge^{p_{i}-1} W^{*} \mid v \in W\right\} \\
& E_{\Phi_{0}}^{k}(W)=\left\{\alpha \wedge i_{v} \Phi_{0} \in \bigoplus_{i=1}^{\ell} \bigwedge^{p_{i}+k-1} W^{*} \mid \alpha \in \bigwedge^{k} W^{*}, v \in W\right\}
\end{aligned}
$$


for integers $k \geq 2$. Then we have a complex

$$
\left(\sharp_{\Phi_{0}}\right) \quad 0 \rightarrow E_{\Phi_{0}}^{0}(W) \stackrel{\wedge u}{\longrightarrow} E_{\Phi_{0}}^{1}(W) \stackrel{\wedge u}{\longrightarrow} E_{\Phi_{0}}^{2}(W) \stackrel{\wedge u}{\longrightarrow} \cdots
$$

for a form $u \in W^{*}$.

Definition 3.1. An orbit $\mathcal{O}$ is elliptic if the complex $\mathbb{H}_{\Phi_{0}}$ is exact for any nonzero element $u \in W^{*}$ at $E_{\Phi_{0}}^{1}(W)$ and $E_{\Phi_{0}}^{2}(W)$.

We give some examples of elliptic orbits. Now we assume that $W$ is evendimensional, that is, $q=2 n$.

Example 1. The set of all symplectic forms on $W$ is an orbit $\mathcal{O}_{\text {symp, which is }}$ isomorphic to the quotient space $\operatorname{GL}(2 n, \mathbb{R}) / \operatorname{Sp}(2 n, \mathbb{R})$. For any $\Phi_{0} \in \mathcal{O}_{\text {symp }}$, the complex $\left(\sharp_{\Phi_{0}}\right)$ is

$$
0 \rightarrow \bigwedge^{1} W^{*} \stackrel{\wedge u}{\longrightarrow} \bigwedge^{2} W^{*} \stackrel{\wedge u}{\longrightarrow} \bigwedge^{3} W^{*} \stackrel{\wedge u}{\longrightarrow} \cdots
$$

for any element $u \in W^{*}$. Thus the orbit $\mathcal{O}_{\text {symp }}$ is elliptic.

Example 2. A non-zero complex $n$-form $\Omega \in \bigwedge^{n} \otimes \mathbb{C}$ is called an $\mathrm{SL}_{n}(\mathbb{C})$ structure on $W$ if the form $\Omega$ satisfies

$$
W \otimes \mathbb{C}=\operatorname{Ker} \Omega \oplus \overline{\operatorname{Ker} \Omega}
$$

where Ker $\Omega$ denotes the space $\left\{v \in W \otimes \mathbb{C} \mid i_{v} \Omega=0\right\}$. We remark that an $\mathrm{SL}_{n}(\mathbb{C})$ structure $\Omega$ induces a complex structure $J_{\Omega}$ on $W$ defined by

$$
J_{\Omega}(v)= \begin{cases}-\sqrt{-1} v & \text { for } v \in \operatorname{Ker} \Omega \\ \sqrt{-1} v & \text { for } v \in \overline{\operatorname{Ker} \Omega}\end{cases}
$$

Then $\Omega$ is an $(n, 0)$-form with respect to the complex structure $J_{\Omega}$. Let $\mathcal{O}_{\mathrm{SL}}$ be the set of $\mathrm{SL}_{n}(\mathbb{C})$ structures on $W$. Then it turns out that $\mathcal{O}_{\mathrm{SL}}$ is an orbit such that

$$
\mathcal{O}_{\mathrm{SL}}=\mathrm{GL}(2 n, \mathbb{R}) / \mathrm{SL}(n, \mathbb{C}) .
$$

For any $\Phi_{0} \in \mathcal{O}_{\mathrm{SL}}$, the complex $\left(\sharp_{\Phi_{0}}\right)$ is

$$
0 \rightarrow \bigwedge^{n-1,0} W^{*} \stackrel{\wedge u}{\longrightarrow} \bigwedge^{n, 0} W^{*} \oplus \bigwedge^{n-1,1} W^{*} \stackrel{\wedge u}{\longrightarrow} \bigwedge^{n, 1} W^{*} \oplus \bigwedge^{n-1,2} W^{*} \stackrel{\wedge u}{\longrightarrow} \cdots
$$

for any $u \in W^{*}$. Here we regard the element $u$ as an element of $\bigwedge^{1,0} W^{*} \oplus \bigwedge^{0,1} W^{*}$ such that $\bar{u}=u$. So this orbit $\mathcal{O}_{\mathrm{SL}}$ is elliptic. 
Example 3. A pair $(\Omega, \omega) \in \bigwedge_{\mathrm{B}}^{n} \otimes \mathbb{C} \oplus \bigwedge_{\mathrm{B}}^{2}$ is called a Calabi-Yau structure on $W$ if $\Omega$ is an $\mathrm{SL}_{n}(\mathbb{C})$ structure and $\omega$ is a symplectic structure on $W$ such that

$$
\begin{aligned}
& \Omega \wedge \omega=0, \\
& \Omega \wedge \bar{\Omega}=c_{n} \omega^{n} \neq 0, \\
& \omega\left(\cdot, J_{\Omega} \cdot\right) \text { is positive definite, }
\end{aligned}
$$

where $c_{n}=\frac{1}{n !}(-1)^{n(n-1) / 2}(2 / \sqrt{-1})^{n}$. Let $\mathcal{O}_{\mathrm{CY}}$ be the set of Calabi-Yau structures on $W$. Then $\mathcal{O}_{\mathrm{CY}}$ is an elliptic orbit such that

$$
\mathcal{O}_{\mathrm{CY}}=\mathrm{GL}(2 n, \mathbb{R}) / \mathrm{SU}(n)
$$

(cf. [8, Proposition 4.9]).

\section{§3.2. Transverse calibrations in foliated manifolds}

Let $M$ be a compact manifold of dimension $p+q$ and $\mathcal{F}$ a Riemannian foliation on $M$ of codimension $q$. We consider the completely integrable distribution $F$ associated to $\mathcal{F}$ and the quotient bundle $Q=T M / F$ over $M$. For each $x \in M$, we identify $Q_{x}$ with $W=\mathbb{R}^{q}$. Then, as in Section 3.1, we have an orbit $\mathcal{A}_{\mathcal{O}}\left(Q_{x}\right)=$ $\mathcal{A}_{\mathcal{O}}(W)$ at $x \in M$ for an orbit $\mathcal{O}$. Note that the orbit $\mathcal{A}_{\mathcal{O}}\left(Q_{x}\right) \subset \bigoplus_{i} \wedge^{p_{i}} Q_{x}^{*}$ does not depend on the choice of the identification $h: Q_{x} \simeq W$. Then we can define a $G / H$-bundle $\mathcal{A}_{\mathcal{O}}(M, \mathcal{F})$ by

$$
\mathcal{A}_{\mathcal{O}}(M, \mathcal{F})=\bigcup_{x \in M} \mathcal{A}_{\mathcal{O}}\left(Q_{x}\right) \rightarrow M
$$

Since $\mathcal{A}_{\mathcal{O}}(M, \mathcal{F}) \subset \bigoplus_{i} \bigwedge^{p_{i}} Q^{*}$, we can consider the Lie derivative and the exterior derivative for any section of $\mathcal{A}_{\mathcal{O}}(M, \mathcal{F})$ as a differential form. We denote by $\mathcal{E}_{\mathcal{O}}(M, \mathcal{F})$ the space of sections of $\mathcal{A}_{\mathcal{O}}(M, \mathcal{F})$ which are basic forms:

$$
\mathcal{E}_{\mathcal{O}}(M, \mathcal{F})=\Gamma\left(\mathcal{A}_{\mathcal{O}}(M, \mathcal{F})\right) \cap \bigwedge_{\mathrm{B}}^{*}
$$

Let $\operatorname{Ker} \Phi$ be the space $\left\{v \in T M \mid i_{v} \Phi=0\right\}$ for $\Phi \in \mathcal{E}_{\mathcal{O}}(M, \mathcal{F})$.

Definition 3.2. A section $\Phi \in \mathcal{E}_{\mathcal{O}}(M, \mathcal{F})$ is called a transverse calibration associated with the orbit $\mathcal{O}$ if $\Phi$ is a closed form such that $\operatorname{Ker} \Phi=F$.

We denote by $\widetilde{\mathfrak{M}}_{\mathcal{O}}(M, \mathcal{F})$ the space of transverse calibrations associated with $\mathcal{O}$. The group $\operatorname{Diff}(M, \mathcal{F})$ acts on $\widetilde{\mathfrak{M}}_{\mathcal{O}}(M, \mathcal{F})$ by pull-back. Given a transverse calibration $\Phi \in \widetilde{\mathfrak{M}}_{\mathcal{O}}(M, \mathcal{F})$, we can consider the vector spaces $E_{\Phi_{x}}^{k}\left(Q_{x}\right)$ at each point $x \in M$, and define vector bundles

$$
E_{\Phi}^{k}(M, \mathcal{F})=\bigcup_{x \in M} E_{\Phi_{x}}^{k}\left(Q_{x}\right) \rightarrow M
$$


for integers $k \geq 0$. Each bundle $E_{\Phi}^{k}(M, \mathcal{F})$ is a basic bundle since its associated principal bundle is that of the normal bundle $Q^{*}$. It follows that a section $i_{v} \Phi \in$ $\Gamma\left(E_{\Phi}^{0}(M, \mathcal{F})\right)$ is basic if and only if $v \in \Gamma(Q)$ is a basic section from $\operatorname{Ker} \Phi=F$ and $L_{w}\left(i_{v} \Phi\right)=i_{L_{w} v} \Phi$ for any $w \in \Gamma(F)$. Hence we have

$$
\begin{aligned}
& \Gamma_{\mathrm{B}}\left(E_{\Phi}^{0}(M, \mathcal{F})\right)=\left\{i_{v} \Phi \in \bigoplus_{i=1}^{\ell} \bigwedge^{p_{i}-1} Q^{*} \mid v \in \Gamma_{\mathrm{B}}(Q)\right\}, \\
& \Gamma_{\mathrm{B}}\left(E_{\Phi}^{1}(M, \mathcal{F})\right)=\left\{\hat{\rho}_{\xi} \Phi \in \bigoplus_{i=1}^{\ell} \bigwedge^{p_{i}} Q^{*} \mid \xi \in \Gamma_{\mathrm{B}}(\operatorname{End}(Q))\right\} .
\end{aligned}
$$

We introduce the graded vector spaces $E_{\Phi}(M, \mathcal{F})=\bigoplus_{k} E_{\Phi}^{k}(M, \mathcal{F})$. For simplicity, we shall denote by $\Gamma_{\mathrm{B}}\left(E^{k}\right)$ and $\Gamma_{\mathrm{B}}(E)$ the spaces $\Gamma_{\mathrm{B}}\left(E_{\Phi}^{k}(M, \mathcal{F})\right)$ and $\Gamma_{\mathrm{B}}\left(E_{\Phi}(M, \mathcal{F})\right)$, respectively.

Proposition 3.1. The module $\Gamma_{\mathrm{B}}(E)$ is a differential graded module in $\bigoplus_{k}\left(\bigoplus_{i} \wedge_{\mathrm{B}}^{p_{i}+k-1}\right)$ with respect to the derivative $d_{\mathrm{B}}$, where $d_{\mathrm{B}}$ is the exterior derivative $d$ restricted to the space of basic forms.

Proof. We prove that $d_{\mathrm{B}} a \in \Gamma_{\mathrm{B}}\left(E^{k}\right)$ for all $a \in \Gamma_{\mathrm{B}}\left(E^{k-1}\right)$. To show this, it is sufficient to prove that $d_{\mathrm{B}} i_{v} \Phi \in \Gamma_{\mathrm{B}}\left(E^{1}\right)$ for any element $i_{v} \Phi \in \Gamma_{\mathrm{B}}\left(E^{0}\right)$, since $\Gamma_{\mathrm{B}}(E)$ is generated by $\Gamma_{\mathrm{B}}\left(E^{0}\right)$. The basic vector field $v$ induces a one-parameter transformation $\left\{f_{t}\right\}$ such that each $f_{t}$ is an element of $\operatorname{Diff}(M, \mathcal{F})$. Then it follows from $d \Phi=0$ that

$$
d i_{v} \Phi=L_{v} \Phi=\left.\frac{d}{d t} f_{t}^{*} \Phi\right|_{t=0} .
$$

Since $f_{t}^{*} \Phi$ is in $\mathcal{E}_{\mathcal{O}}(M, \mathcal{F}),\left.\frac{d}{d t} f_{t}^{*} \Phi\right|_{t=0}$ lies in the tangent space of $\mathcal{E}_{\mathcal{O}}(M, \mathcal{F})$ at $\Phi$, which is the space $\Gamma_{\mathrm{B}}\left(E^{1}\right)$. This implies that $d i_{v} \Phi=\left.\frac{d}{d t} f_{t}^{*} \Phi\right|_{t=0} \in \Gamma_{\mathrm{B}}\left(E^{1}\right)$.

Thus we obtain a complex

$$
0 \rightarrow \Gamma_{\mathrm{B}}\left(E^{0}\right) \stackrel{d_{0}}{\longrightarrow} \Gamma_{\mathrm{B}}\left(E^{1}\right) \stackrel{d_{1}}{\longrightarrow} \Gamma_{\mathrm{B}}\left(E^{2}\right) \stackrel{d_{2}}{\longrightarrow} \cdots
$$

where $d_{i}=\left.d_{\mathrm{B}}\right|_{E^{i}}$ for each $i$. The complex $\underset{\mathbb{A}_{\Phi}}{ }$ is a subcomplex of the basic de Rham complex:

$$
\begin{gathered}
0 \rightarrow \Gamma_{\mathrm{B}}\left(E^{0}\right) \stackrel{d_{0}}{\longrightarrow} \Gamma_{\mathrm{B}}\left(E^{1}\right) \stackrel{d_{1}}{\longrightarrow} \Gamma_{\mathrm{B}}\left(E^{2}\right) \stackrel{d_{2}}{\longrightarrow} \cdots \\
\quad \downarrow \\
\cdots \\
\cdots
\end{gathered}
$$

We denote by $H^{k}\left(\sharp_{\Phi}\right)$ the cohomology groups of the complex $\mathbb{\sharp}_{\Phi}$ :

$$
H^{k}\left(\sharp_{\Phi}\right)=\left\{\alpha \in \Gamma_{\mathrm{B}}\left(E^{k}\right) \mid d_{k} \alpha=0\right\} /\left\{d_{k-1} \beta \in \Gamma_{\mathrm{B}}\left(E^{k}\right) \mid \beta \in \Gamma_{\mathrm{B}}\left(E^{k-1}\right)\right\} .
$$

Then, for each $k \geq 0$, we define $p^{k}$ as the map in cohomology induced by the chain map in 3.2 :

$$
p^{k}: H^{k}\left(\sharp_{\Phi}\right) \rightarrow \bigoplus_{i} H_{\mathrm{B}}^{p_{i}+k-1}(M) .
$$


If $\mathcal{O}$ is an elliptic orbit, then the complex $\left(\sharp_{\Phi}\right)$ is transverse elliptic at $\Gamma_{\mathrm{B}}\left(E^{1}\right)$ and $\Gamma_{\mathrm{B}}\left(E^{2}\right)$. Hence the cohomology groups $H^{k}\left(\sharp_{\Phi}\right)$ for $k=1,2$ are finite-dimensional from Proposition 2.1 .

\section{§4. Deformations of transverse calibrations}

We suppose that $\mathcal{F}$ is a Riemannian foliation on a compact manifold $M$ and $\widetilde{\mathfrak{M}}_{\mathcal{O}}(M, \mathcal{F})$ is the set of transverse calibrations associated with an orbit $\mathcal{O}$ on $(M, \mathcal{F})$. Let $\mathcal{H}$ be the space of all closed forms on $M$ and let $\mathcal{E}$ be the space of sections $\Phi$ of $\mathcal{E}_{\mathcal{O}}(M, \mathcal{F})$ satisfying $\operatorname{Ker} \Phi=F$. The space $\widetilde{\mathfrak{M}}_{\mathcal{O}}(M, \mathcal{F})$ is the intersection $\mathcal{H} \cap \mathcal{E}$. Hence we can regard the infinitesimal tangent space of $\widetilde{\mathfrak{M}}_{\mathcal{O}}(M, \mathcal{F})$ as $\mathcal{H} \cap T_{\Phi} \mathcal{E}\left(=\mathcal{H} \cap \Gamma_{\mathrm{B}}\left(E^{1}\right)\right)$. We will investigate whether the infinitesimal tangent space agrees with the tangent space of actual deformations.

Definition 4.1. A transverse calibration $\Phi \in \widetilde{\mathfrak{M}}_{\mathcal{O}}(M, \mathcal{F})$ is unobstructed if for any $\alpha \in \mathcal{H} \cap \Gamma_{\mathrm{B}}\left(E^{1}\right)$ there exists a smooth curve $\Phi_{t}$ in $\widetilde{\mathfrak{M}}_{\mathcal{O}}(M, \mathcal{F})$ such that

$$
\left.\frac{d}{d t} \Phi_{t}\right|_{t=0}=\alpha
$$

We provide the following criterion for the unobstructedness of transverse calibrations:

Theorem 4.1. Let $\mathcal{O}$ be an elliptic orbit. If $p^{2}: H^{2}\left(\sharp_{\Phi}\right) \rightarrow \bigoplus_{i} H_{\mathrm{B}}^{p_{i}+1}(M)$ is injective for $\Phi \in \widetilde{\mathfrak{M}}_{\mathcal{O}}(M, \mathcal{F})$, then $\Phi$ is unobstructed.

Proof. We apply the argument of Section 2 in 8 to basic differential forms. Define a linear operator $\operatorname{Ad}_{a}^{1} d: \bigwedge_{\mathrm{B}}^{*} \rightarrow \bigwedge_{\mathrm{B}}^{*}$ for $a \in \Gamma_{\mathrm{B}}(\operatorname{End}(Q))$ as the commutator $\left[d, \hat{\rho}_{a}\right]=d \circ \hat{\rho}_{a}-\hat{\rho}_{a} \circ d:$

$$
\operatorname{Ad}_{a}^{1} d=\left[d, \hat{\rho}_{a}\right]
$$

Inductively, we define linear operators $\operatorname{Ad}_{a}^{\ell} d: \bigwedge_{\mathrm{B}}^{*} \rightarrow \bigwedge_{\mathrm{B}}^{*}$ for $\ell \geq 2$ as

$$
\operatorname{Ad}_{a}^{\ell} d=\left[\operatorname{Ad}_{a}^{\ell-1} d, \hat{\rho}_{a}\right]
$$

for $a \in \Gamma_{\mathrm{B}}(\operatorname{End}(Q))$. Let $\left(e^{\operatorname{Ad}_{a}}\right) d$ be the formal power series defined by

$$
\left(e^{\operatorname{Ad}_{a}}\right) d=\sum_{\ell=0}^{\infty} \frac{1}{\ell !}\left(\operatorname{Ad}_{a}^{\ell} d\right) .
$$

The action $\rho_{e^{a}}$ of the exponential $e^{a} \in \Gamma_{\mathrm{B}}(\mathrm{GL}(Q))$ is written as

$$
\rho_{e^{a}}=1+\hat{\rho}_{a}+\frac{1}{2 !} \hat{\rho}_{a} \hat{\rho}_{a}+\frac{1}{3 !} \hat{\rho}_{a} \hat{\rho}_{a} \hat{\rho}_{a}+\cdots
$$


Then

$$
\rho_{e^{-a}} d \rho_{e^{a}}=\left(e^{\operatorname{Ad}_{a}}\right) d
$$

for any section $a \in \Gamma_{\mathrm{B}}(\operatorname{End}(Q))$.

We start to show the theorem. Suppose that $\alpha$ is in $\mathcal{H} \cap \Gamma_{\mathrm{B}}\left(E^{1}\right)$. Then there exists an element $a_{1} \in \Gamma_{\mathrm{B}}(\operatorname{End}(Q))$ such that $\alpha=\hat{\rho}_{a_{1}} \Phi$ by the definition of $\Gamma_{\mathrm{B}}\left(E^{1}\right)$. Now we consider formal power series $a(t)$ in $t$ :

$$
a(t)=a_{1} t+\frac{1}{2 !} a_{2} t^{2}+\frac{1}{3 !} a_{3} t^{3}+\cdots \in \Gamma_{\mathrm{B}}(\operatorname{End}(Q))[[t]]
$$

where each $a_{k}$ is a basic section of $\operatorname{End}(Q)$. We define an element $\Phi_{t}$ of $\mathcal{E}$ as

$$
\Phi_{t}=\rho_{e^{a(t)}} \Phi
$$

for $e^{a(t)} \in \Gamma_{\mathrm{B}}(\mathrm{GL}(Q))[[t]]$. Then $\Phi_{t}$ satisfies $\left.\frac{d}{d t} \Phi_{t}\right|_{t=0}=\alpha$. Hence it is sufficient to show that there exists an element $a(t)$ such that $\Phi_{t}$ is a closed form, that is,

$$
d \rho_{e^{a(t)}} \Phi=0 .
$$

Equation 4.3 is equivalent to

$$
\left(e^{\operatorname{Ad}_{a(t)}} d\right) \Phi=0
$$

by 4.2 . Now we define $\left(\left(e^{\operatorname{Ad}_{a(t)}} d\right) \Phi\right)_{[k]}$ to be the $k$-th homogeneous part of $\left(e^{\operatorname{Ad}_{a(t)}} d\right) \Phi$ with respect to $t$. Then 4.4 reduces to the system of infinitely many equations

$$
\left(\left(e^{\operatorname{Ad}_{a(t)}} d\right) \Phi\right)_{[k]}=0 .
$$

We determine the coefficients $a_{k}$ by induction on $k$, in order to obtain a solution $a(t)$. First, the element $a_{1}$ satisfies $d \hat{\rho}_{a_{1}} \Phi=0$ since $\hat{\rho}_{a_{1}} \Phi=\alpha \in \mathcal{H}$. It follows from the condition $d \Phi=0$ that $\left(\left(e^{\operatorname{Ad}_{a(t)}} d\right) \Phi\right)_{[1]}=d \Phi+\left[d, \hat{\rho}_{a_{1}}\right] \Phi=0$. Next, we assume that $a_{1}, \ldots, a_{k-1}$ satisfy $\left(\left(e^{\operatorname{Ad}_{a(t)}} d\right) \Phi\right)_{[j]}=0$ for $j \leq k-1$. We define a form $\mathrm{Ob}\left(a_{1}, \ldots, a_{k-1}\right)$ by

$$
\operatorname{Ob}\left(a_{1}, \ldots, a_{k-1}\right)=\left(\sum_{\ell=2}^{k} \frac{1}{\ell !}\left(\operatorname{Ad}_{a(t)}^{\ell} d\right) \Phi\right)_{[k]} .
$$

For simplicity, we denote by $\mathrm{Ob}_{k}$ the obstruction form $\mathrm{Ob}\left(a_{1}, \ldots, a_{k-1}\right)$. Then $\mathrm{Ob}_{k}$ is a section of $\Gamma_{\mathrm{B}}\left(E^{2}\right)$ and satisfies

$$
\left(\left(e^{\operatorname{Ad}_{a(t)}} d\right) \Phi\right)_{[k]}=\mathrm{Ob}_{k}+\frac{1}{k !} d \hat{\rho}_{a_{k}} \Phi .
$$


From the induction hypothesis, we have $\left(d \rho_{e^{a(t)}} \Phi\right)_{[k]}=\left(\left(e^{\operatorname{Ad}_{a(t)}} d\right) \Phi\right)_{[k]}$. Thus $\mathrm{Ob}_{k}$ is $d$-exact and $d_{2}$-closed. We can consider the cohomology class $\left[\mathrm{Ob}_{k}\right]$ in $H^{2}\left(\sharp_{\Phi}\right)$ and call it the $k$-th obstruction class. In the case of $\left(\left(e^{\operatorname{Ad}_{a(t)}} d\right) \Phi\right)_{[k]}=0$, one has $\left[\mathrm{Ob}_{k}\right]=\left[\frac{1}{k !} d \hat{\rho}_{a_{k}} \Phi\right]=0$ in $H^{2}\left(\sharp_{\Phi}\right)$. Conversely, if the class $\left[\mathrm{Ob}_{k}\right]$ is zero, then $a_{k}$ may be found so that $a(t)$ satisfies the equation $\left(\left(e^{\operatorname{Ad}_{a(t)}} d\right) \Phi\right)_{[k]}=0$. In fact, by applying basic Hodge theory to the basic elliptic complex $\left(\sharp_{\Phi}\right)$, we can take an element $a_{k}$ such that

$$
\frac{1}{k !} \hat{\rho}_{a_{k}} \Phi=-d_{1}^{*} G_{\sharp}\left(\mathrm{Ob}_{k}\right)
$$

where $G_{\sharp}$ and $d_{1}^{*}$ are the Green's operator and the adjoint operator of $d_{1}$, respectively.

If the map $p^{2}: H^{2}\left(\sharp_{\Phi}\right) \rightarrow \bigoplus_{i} H_{\mathrm{B}}^{p_{i}+1}(M)$ is injective, then the obstruction class $\left[\mathrm{Ob}_{k}\right] \in H^{2}\left(\sharp_{\Phi}\right)$ vanishes for all $k$ since $\mathrm{Ob}_{k}$ is $d$-exact. By the above argument, the elements $a_{k}$ defined by (4.6) satisfy 4.5 for all $k \geq 1$. Hence we obtain a solution $a(t)$ of 4.3 as a formal power series. Moreover, this formal power series converges uniformly in the Sobolev norm $\|\cdot\|_{s}$.

We discuss the regularity of the solution $a(t)$ constructed above. We recall that the element $a_{1}$ satisfies $d \hat{\rho}_{a_{1}} \Phi=0$ as an initial condition and the coefficients $a_{k}$ for $k \geq 2$ are defined by (4.6). Hence the power series $a(t)$ satisfies

$$
d_{0}^{*} \hat{\rho}_{a(t)} \Phi=d_{0}^{*} \hat{\rho}_{a_{1}} \Phi
$$

since $d_{0}^{*} \hat{\rho}_{a_{k}} \Phi=0$ for $k \geq 2$. Moreover, we have

$$
d \hat{\rho}_{a(t)} \Phi+\sum_{\ell=2}^{\infty} \frac{1}{\ell} d \hat{\rho}_{a(t)}^{\ell} \Phi=0
$$

from $d \rho_{e^{a(t)}} \Phi=0$ and 4.1 . Thus $\hat{\rho}_{a(t)} \Phi$ is weak solution of the basic elliptic differential equation

$$
\Delta_{\sharp} \hat{\rho}_{a(t)} \Phi+d_{1}^{*}\left(\sum_{\ell=2}^{\infty} \frac{1}{\ell} d \hat{\rho}_{a(t)}^{\ell} \Phi\right)=d_{0} d_{0}^{*} \hat{\rho}_{a_{1}} \Phi
$$

where $\Delta_{\sharp}$ is the basic Laplace operator $d_{1}^{*} d_{1}+d_{0} d_{0}^{*}$. Elliptic regularity implies that the solution $\hat{\rho}_{a(t)} \Phi$ is smooth for a smooth element $\hat{\rho}_{a_{1}} \Phi$. Hence the form $\rho_{e^{a(t)}} \Phi$ is also smooth.

\section{$\S 5$. A generalization of Moser's stability theorem}

Let $M$ be a compact manifold of dimension $p+q$ and $\mathcal{F}$ a Riemannian foliation on $M$ of codimension $q$. Let $\widetilde{\mathfrak{M}}_{\mathcal{O}}(M, \mathcal{F})$ be the set of transverse calibrations associated with an orbit $\mathcal{O}$ on $(M, \mathcal{F})$. 
We fix a transverse calibration $\Phi \in \widetilde{\mathfrak{M}}_{\mathcal{O}}(M, \mathcal{F})$. Let $E_{\Phi}^{k}$ denote the vector bundle $E_{\Phi}^{k}(M, \mathcal{F})$ defined in Section 3.2. We define a vector bundle $E_{\Phi}^{k, \perp}$ to be the orthogonal complement of $E_{\Phi}^{k}$ in $\bigoplus_{i} \wedge^{p_{i}+k-1} Q^{*}$ with respect to the metric induced by the transverse Riemannian structure on $(M, \mathcal{F})$. We can identify $\Gamma_{\mathrm{B}}\left(E_{\Phi}^{k, \perp}\right)$ with the quotient $\bigoplus_{i} \wedge_{\mathrm{B}}^{p_{i}+k-1} / \Gamma_{\mathrm{B}}\left(E_{\Phi}^{k}\right)$ since each vector bundle $E_{\Phi}^{k, \perp}$ is isomorphic to the quotient bundle $\bigoplus_{i} \bigwedge^{p_{i}+k-1} Q^{*} / E_{\Phi}^{k}$. Then the basic exterior derivative $d_{\mathrm{B}}$ on $\bigoplus_{i} \bigwedge_{\mathrm{B}}^{p_{i}+k-1}$ induces a differential operator $d^{\perp}: \Gamma_{\mathrm{B}}\left(E_{\Phi}^{k, \perp}\right) \rightarrow \Gamma_{\mathrm{B}}\left(E_{\Phi}^{k+1, \perp}\right)$, and we obtain the differential complex

$$
0 \rightarrow \Gamma_{\mathrm{B}}\left(E_{\Phi}^{0, \perp}\right) \stackrel{d^{\perp}}{\longrightarrow} \Gamma_{\mathrm{B}}\left(E_{\Phi}^{1, \perp}\right) \stackrel{d^{\perp}}{\longrightarrow} \Gamma_{\mathrm{B}}\left(E_{\Phi}^{2, \perp}\right) \stackrel{d^{\perp}}{\longrightarrow} \cdots
$$

For simplicity, we shall denote by $E^{k, \perp}$ the bundle $E_{\Phi}^{k, \perp}$. The complex $\sharp_{\Phi}^{\perp}$ is related to the basic de Rham complex as follows:

$$
\begin{aligned}
& \cdots \rightarrow \bigoplus_{i} \bigwedge_{\mathrm{B}}^{p_{i}-1} \stackrel{d_{\mathrm{B}}}{\longrightarrow} \bigoplus_{i} \bigwedge_{\mathrm{B}}^{p_{i}} \stackrel{d_{\mathrm{B}}}{\longrightarrow} \bigoplus_{i} \bigwedge_{\mathrm{B}}^{p_{i}+1} \stackrel{d_{\mathrm{B}}}{\longrightarrow} \cdots \\
& 0 \rightarrow \Gamma_{\mathrm{B}}\left(E^{0, \perp}\right) \stackrel{d^{\perp}}{\longrightarrow} \Gamma_{\mathrm{B}}\left(E^{1, \perp}\right) \stackrel{d^{\perp}}{\longrightarrow} \Gamma_{\mathrm{B}}\left(E^{2, \perp}\right) \stackrel{d^{\perp}}{\longrightarrow} \cdots
\end{aligned}
$$

where the vertical arrows are epimorphisms. We denote by $H^{k}\left(\sharp \frac{\perp}{\Phi}\right)$ the cohomology groups of the complex $\left(\mathbb{H} \frac{1}{\Phi}\right)$ :

$$
H^{k}\left(\sharp_{\Phi}^{\perp}\right)=\left\{\alpha \in \Gamma_{\mathrm{B}}\left(E^{k, \perp}\right) \mid d^{\perp} \alpha=0\right\} /\left\{d^{\perp} \beta \in \Gamma_{\mathrm{B}}\left(E^{k, \perp}\right) \mid \beta \in \Gamma_{\mathrm{B}}\left(E^{k-1, \perp}\right)\right\} .
$$

Then, for each $k \geq 0$, we define $p^{k, \perp}$ as the map in cohomology induced by the chain map in (5.1):

$$
p^{k, \perp}: \bigoplus_{i} H_{\mathrm{B}}^{p_{i}+k-1}(M) \rightarrow H^{k}\left(\sharp_{\Phi}^{\perp}\right) .
$$

We see that there is a relation between the complex $\mathbb{H}_{\Phi}^{\frac{1}{\Phi}}$ and the complex $\#_{\Phi}$ defined in Section 3.2. By the construction of $E^{k, \perp}$ we obtain the commutative diagram

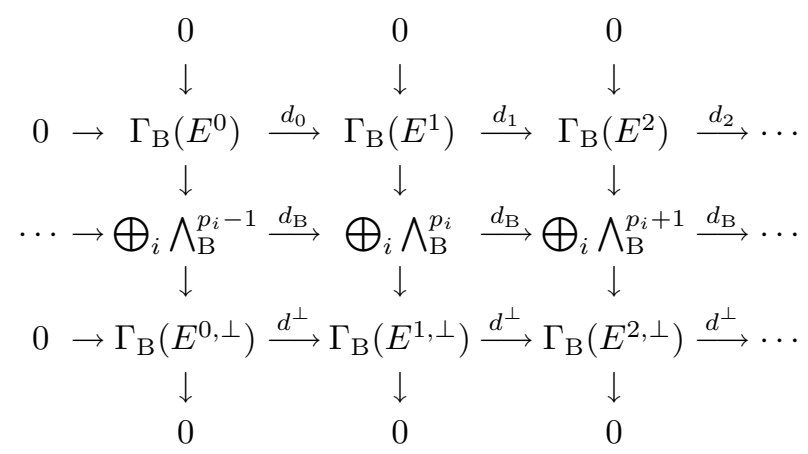

where the columns are exact. By the standard construction of a long exact sequence in cohomology from a short exact sequence of chain complexes, we can easily show 
Lemma 5.1. If the complex $\sharp_{\Phi}$ is transversely elliptic at $\Gamma_{\mathrm{B}}\left(E^{k}\right)$, then the complex $\sharp_{\Phi}^{\perp}$ is also transversely elliptic at $\Gamma_{\mathrm{B}}\left(E^{k-1, \perp}\right)$.

Next, we provide a sufficient condition for the map $p^{1, \perp}$ to be surjective.

Lemma 5.2. If the map $p^{2}: H^{2}\left(\sharp_{\Phi}\right) \rightarrow \bigoplus_{i} H_{\mathrm{B}}^{p_{i}+1}(M)$ is injective, then the map $p^{1, \perp}: \bigoplus_{i} H_{\mathrm{B}}^{p_{i}}(M) \rightarrow H^{1}\left(\sharp \frac{\perp}{\Phi}\right)$ is surjective.

Proof. We define an injection $i$ and a surjection $\pi$ as follows:

$$
0 \rightarrow \Gamma_{\mathrm{B}}\left(E^{k}\right) \stackrel{i}{\rightarrow} \bigoplus_{i} \bigwedge_{\mathrm{B}}^{p_{i}+k-1} \stackrel{\pi}{\rightarrow} \Gamma_{\mathrm{B}}\left(E^{k, \perp}\right) \rightarrow 0
$$

for each $k \geq 0$. Now we consider the diagram

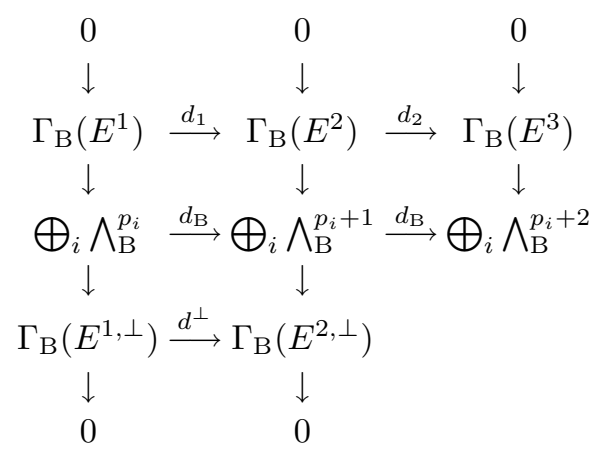

Suppose $\left[\phi^{\perp}\right] \in H^{1}\left(\sharp_{\Phi}^{\perp}\right)$ where $\phi^{\perp}$ is an element of $\Gamma_{\mathrm{B}}\left(E^{1, \perp}\right)$ with $d^{\perp} \phi^{\perp}=0$. Then we can choose an element $\phi^{\prime} \in \bigoplus_{i} \bigwedge_{\mathrm{B}}^{p_{i}}$ such that $\pi\left(\phi^{\prime}\right)=\phi^{\perp}$. This $\phi^{\prime}$ satisfies $\pi\left(d_{\mathrm{B}} \phi^{\prime}\right)=d^{\perp} \pi\left(\phi^{\prime}\right)=0$. From the exactness of $\Gamma_{\mathrm{B}}\left(E^{2}\right) \rightarrow \bigoplus_{i} \bigwedge_{\mathrm{B}}^{p_{i}+1} \rightarrow \Gamma_{\mathrm{B}}\left(E^{2, \perp}\right)$, there exists an element $\psi \in \Gamma_{\mathrm{B}}\left(E^{2}\right)$ with $i(\psi)=d_{\mathrm{B}} \phi^{\prime}$. Then $\psi$ is $d_{2}$-closed, and the class $[\psi] \in H^{2}\left(\sharp_{\Phi}\right)$ is in the kernel of $p^{2}$. So we can take an element $\phi^{\prime \prime} \in \Gamma_{\mathrm{B}}\left(E^{1}\right)$ with $d_{1} \phi^{\prime \prime}=\psi$ since $p^{2}$ is injective. Now we define $\phi$ by $\phi^{\prime}-i\left(\phi^{\prime \prime}\right) \in \bigoplus_{i} \bigwedge_{\mathrm{B}}^{p_{i}+1}$. Then it is easy to see that $d_{\mathrm{B}} \phi=0$ and $\pi(\phi)=\phi^{\perp}$. Thus we obtain the class $[\phi] \in \bigoplus_{i} H_{\mathrm{B}}^{p_{i}}(M)$ satisfying $p^{1, \perp}([\phi])=\left[\phi^{\perp}\right]$, and this finishes the proof.

We now show that the dimension of $H^{1}\left(\sharp_{\Phi}\right)$ is invariant under deformations of $\Phi \in \widetilde{\mathfrak{M}}_{\mathcal{O}}(M, \mathcal{F})$ :

Proposition 5.1. Let $\mathcal{O}$ be an elliptic orbit and $\left\{\Phi_{t}\right\}_{t \in[0,1]}$ a smooth family of $\widetilde{\mathfrak{M}}_{\mathcal{O}}(M, \mathcal{F})$. If the maps $p^{1}$ and $p^{2}$ are injective for each $t \in[0,1]$, then $\operatorname{dim} H^{1}\left(\sharp_{\Phi_{t}}\right)$ is independent of $t \in[0,1]$.

Proof. The complex $\left(\sharp_{\Phi_{t}}\right)$ is transversely elliptic at $E_{\Phi_{t}}^{1}$ by the definition of the elliptic orbit $\mathcal{O}$. The complex $\left(\sharp_{\Phi_{t}}^{\perp}\right)$ is also transversely elliptic at $E_{\Phi_{t}}^{1, \perp}$ by Lemma 5.1 . 
Therefore, each of the cohomology groups $H^{1}\left(\sharp \Phi_{t}\right)$ and $H^{1}\left(\sharp \Psi_{\Phi_{t}}\right)$ is the kernel of a transversely elliptic operator. Hence the dimensions $\operatorname{dim} H^{1}\left(\sharp_{\Phi_{t}}\right)$ and $\operatorname{dim} H^{1}\left(\sharp_{\Phi_{t}}^{\perp}\right)$ are upper semicontinuous in $t$.

We have the exact sequence

$$
0 \rightarrow H^{1}\left(\sharp_{\Phi_{t}}\right) \rightarrow \bigoplus_{i} H_{\mathrm{B}}^{p_{i}}(M) \rightarrow H^{1}\left(\sharp_{\Phi_{t}}^{\perp}\right) \rightarrow 0
$$

for each $t \in[0,1]$ by using Lemma 5.2 and the assumptions on $p^{1}$ and $p^{2}$. This sequence yields

$$
\operatorname{dim} H^{1}\left(\sharp_{\Phi_{t}}\right)=\sum_{i} \operatorname{dim} H_{\mathrm{B}}^{p_{i}}(M)-\operatorname{dim} H^{1}\left(\sharp_{\Phi_{t}}^{\perp}\right)
$$

for each $t \in[0,1]$. From 5.2 and the upper semicontinuity of $\operatorname{dim} H^{1}\left(\sharp_{\Phi_{t}}\right)$ it follows that $\operatorname{dim} H^{1}\left(\sharp_{\Phi_{t}}\right)$ is lower semicontinuous. On the other hand, it is upper semicontinuous. Therefore it is independent of $t$ in a small interval, and hence in the whole $[0,1]$ by compactness. This completes the proof.

Let $\operatorname{Diff}_{0}(M, \mathcal{F})$ denote the identity component of $\operatorname{Diff}(M, \mathcal{F})$. We provide a generalization of Moser's stability theorem:

Theorem 5.1. Let $\mathcal{O}$ be an elliptic orbit and $\left\{\Phi_{t}\right\}_{t \in[0,1]}$ a smooth family of $\widetilde{\mathfrak{M}}_{\mathcal{O}}(M, \mathcal{F})$ with the same basic cohomology class $\left[\Phi_{t}\right]=\left[\Phi_{0}\right] \in \bigoplus_{i} H_{\mathrm{B}}^{p_{i}}(M)$. If the maps $p^{1}$ and $p^{2}$ are injective for each $t \in[0,1]$, then there exists a smooth family $\left\{f_{t}\right\}_{t \in[0,1]}$ of $\operatorname{Diff}_{0}(M, \mathcal{F})$ such that $f_{0}=\operatorname{id}_{M}$ and $f_{t}^{*} \Phi_{t}=\Phi_{0}$.

Proof. By assumption we may choose an element $A_{t} \in \bigoplus_{i} \wedge_{\mathrm{B}}^{p_{i}}$ with smooth dependence on $t$ such that

$$
\Phi_{t}-\Phi_{0}=d A_{t}
$$

for each $t \in[0,1]$. It suffices to find a smooth family $f_{t} \in \operatorname{Diff}_{0}(M, \mathcal{F})$ such that

$$
\frac{d}{d t}\left(f_{t}^{*} \Phi_{t}\right)=0
$$

Note that $\left(f_{t}^{-1}\right)^{*} \dot{f}_{t}^{*} \Phi_{t}$ is the infinitesimal transformation $L_{v_{t}} \Phi_{t}$ for some basic vector field $v_{t}$. So 5.3 is equivalent to

$$
L_{v_{t}} \Phi_{t}+d \dot{A}_{t}=0 .
$$

Hence the proof reduces to finding a smooth family of basic vector fields $v_{t} \in \Gamma_{\mathrm{B}}(Q)$ with $L_{v_{t}} \Phi_{t}=-d \dot{A}_{t}$. Now the class $\left[d \dot{A}_{t}\right]$ of $H^{1}\left(\sharp_{\Phi_{t}}\right)$ vanishes since $p^{1}: H^{1}\left(\sharp_{\Phi_{t}}\right) \rightarrow$ $\bigoplus_{i} H_{\mathrm{B}}^{p_{i}}(M)$ is injective. Thus we can define an element $B_{t}$ of $\Gamma_{\mathrm{B}}\left(E_{\Phi_{t}}^{0}\right)$ as

$$
B_{t}=-d_{0}^{*} G_{\sharp \Phi_{t}} d \dot{A}_{t}
$$


where $d_{0}^{*}$ and $G_{\sharp_{\Phi_{t}}}$ denote the adjoint operator of $d_{0}$ and Green's operator at $\Gamma_{\mathrm{B}}\left(E_{\Phi_{t}}^{1}\right)$ of the complex $\left(\sharp \Phi_{t}\right)$, respectively. Note that $\operatorname{dim} H^{1}\left(\sharp_{\Phi_{t}}\right)$ is independent of $t$ from Proposition 5.1 so the families $d \dot{A}_{t}=\dot{\Phi}_{t}$ and $G_{\sharp_{\Phi_{t}}}$ are smooth in $t \in[0,1]$. Hence there exists a smooth family $v_{t} \in \Gamma_{\mathrm{B}}(Q)$ such that $B_{t}=i_{v_{t}} \Phi_{t}$ by the definition of $\Gamma_{\mathrm{B}}\left(E_{\Phi_{t}}^{0}\right)$. Then $L_{v_{t}} \Phi_{t}=d B_{t}=-d \dot{A}_{t}$. We thus obtain a smooth family $\left\{f_{t}\right\}_{t \in[0,1]}$ in $\operatorname{Diff}_{0}(M, \mathcal{F})$ satisfying $[5.3$, which completes the proof.

\section{§6. Applications}

We assume that $M$ is a compact manifold with a Riemannian foliation $\mathcal{F}$ of codimension $2 n$. Let $F$ denote the integrable distribution induced by $\mathcal{F}$.

\section{§6.1. Transverse symplectic structures}

A transverse symplectic foliation $\mathcal{F}$ on $M$ is characterized by the following structure.

Definition 6.1. A real 2-form $\omega \in \Lambda^{2}$ is called a transverse symplectic structure on $(M, \mathcal{F})$ if $\omega$ is a basic form on $(M, \mathcal{F})$ such that $d \omega=0$ and $\omega^{n} \neq 0$.

Let $\widetilde{\mathfrak{M}}_{\text {symp }}(M, \mathcal{F})$ be the set of transverse symplectic structures on $(M, \mathcal{F})$. A transverse symplectic structure $\omega$ is a transverse calibration associated with the orbit $\mathcal{O}_{\text {symp }}$. Hence we can regard $\widetilde{\mathfrak{M}}_{\text {symp }}(M, \mathcal{F})$ as the set of transverse calibrations associated with $\mathcal{O}_{\text {symp }}$.

Lemma 6.1. For any $\omega \in \widetilde{\mathfrak{M}}_{\mathrm{symp}}(M, \mathcal{F})$, the maps $p^{k}: H^{k}\left(\sharp_{\omega}\right) \rightarrow H_{\mathrm{B}}^{k+1}(M)$ are injective for $k \geq 1$.

Proof. For a transverse symplectic structure $\omega \in \widetilde{\mathfrak{M}}_{\text {symp }}(M, \mathcal{F})$, it is known that $\Gamma_{\mathrm{B}}\left(E_{\omega}^{k}\right)=\bigwedge_{\mathrm{B}}^{k+1}$ for $k \geq 0$. Therefore we obtain the cohomology group $H^{k}\left(\sharp_{\omega}\right)=$ $H_{\mathrm{B}}^{k+1}(M)$ for each $k \geq 1$, so each $p^{k}: H^{k}\left(\sharp_{\omega}\right) \rightarrow H_{\mathrm{B}}^{k+1}(M)$ is an isomorphism.

Observe that a transverse symplectic structure $\omega$ on $(M, \mathcal{F})$ is a presymplectic form on $M$ such that $\operatorname{Ker} \omega=F$. By applying Theorem 5.1 to the elliptic orbit $\mathcal{O}_{\text {symp }}$, we obtain Moser's stability theorem for presymplectic forms:

Theorem 6.1. Let $\left\{\omega_{t}\right\}_{t \in[0,1]}$ be a smooth family of presymplectic forms on $M$ with $\operatorname{Ker} \omega_{t}=F$ and the same basic cohomology class $\left[\omega_{t}\right]=\left[\omega_{0}\right] \in H_{\mathrm{B}}^{2}(M)$ for all $t \in[0,1]$. Then there exists a smooth family $\left\{f_{t}\right\}_{t \in[0,1]}$ in $\operatorname{Diff}_{0}(M, \mathcal{F})$ such that $f_{0}=\mathrm{id}_{M}$ and $f_{t}^{*} \omega_{t}=\omega_{0}$. 


\section{$\S 6.2$. Transverse $\mathrm{SL}_{n}(\mathbb{C})$ structures}

Definition 6.2. A nowhere vanishing complex $n$-form $\Omega \in \bigwedge^{n} \otimes \mathbb{C}$ is called a transverse $\mathrm{SL}_{n}(\mathbb{C})$ structure on $(M, \mathcal{F})$ if $\Omega$ is a basic form such that $d \Omega=0$ and

$$
Q \otimes \mathbb{C}=\operatorname{Ker} \Omega / F \oplus \overline{\operatorname{Ker} \Omega / F}
$$

where $\operatorname{Ker} \Omega=\left\{v \in T M \otimes \mathbb{C} \mid i_{v} \Omega=0\right\}$.

A transverse $\mathrm{SL}_{n}(\mathbb{C})$ structure $\Omega$ induces a complex structure $J_{\Omega}$ of $Q$ such that $\Omega$ is an $(n, 0)$-basic form on $(M, \mathcal{F})$ (see Example 2). Then we can check that $d \theta \in \bigwedge_{\mathrm{B}}^{2,0} \oplus \bigwedge_{\mathrm{B}}^{1,1}$ for any $\theta \in \bigwedge_{\mathrm{B}}^{1,0}$ because $(d \theta) \wedge \Omega=0$. It follows from the Remark before Definition 2.7 that $J_{\Omega}$ is a transverse complex structure on $(M, \mathcal{F})$. Hence $\left(\mathcal{F}, J_{\Omega}\right)$ is a transverse holomorphic foliation on $M$. Let $\widetilde{\mathfrak{M}}_{\mathrm{SL}}(M, \mathcal{F})$ be the space of transverse $\mathrm{SL}_{n}(\mathbb{C})$ structures on $(M, \mathcal{F})$. Any element $\Omega \in \widetilde{\mathfrak{M}}_{\mathrm{SL}}(M, \mathcal{F})$ induces a transverse calibration associated with the orbit $\mathcal{O}_{\mathrm{SL}}$, and conversely. Thus, we can identify $\widetilde{\mathfrak{M}}_{\mathrm{SL}}(M, \mathcal{F})$ with the set $\widetilde{\mathfrak{M}}_{\mathcal{O}_{\mathrm{SL}}}(M, \mathcal{F})$ of transverse calibrations associated with the orbit $\mathcal{O}_{\mathrm{SL}}$. We recall that the orbit $\mathcal{O}_{\mathrm{SL}}$ is elliptic. For $\Omega \in \widetilde{\mathfrak{M}}_{\mathrm{SL}}(M, \mathcal{F})$ the complex $(\sharp \Omega)$ is

$$
0 \rightarrow \bigwedge_{\mathrm{B}}^{n-1,0} \stackrel{d_{0}}{\longrightarrow} \bigwedge_{\mathrm{B}}^{n, 0} \oplus \bigwedge_{\mathrm{B}}^{n-1,1} \stackrel{d_{1}}{\longrightarrow} \bigwedge_{\mathrm{B}}^{n, 1} \oplus \bigwedge_{\mathrm{B}}^{n-1,2} \stackrel{d_{2}}{\longrightarrow} \cdots
$$

Unfortunately, the maps $p^{1}$ and $p^{2}$ are not always injective for $\Omega \in \widetilde{\mathfrak{M}}_{\mathrm{SL}}(M, \mathcal{F})$. However, we obtain

Proposition 6.1. If $\left(\mathcal{F}, J_{\Omega}\right)$ is a transverse Kähler foliation, then $\Omega$ is unobstructed.

Proof. We suppose that $\Omega \in \widetilde{\mathfrak{M}}_{\mathrm{SL}}(M, \mathcal{F})$ is such that $\left(\mathcal{F}, J_{\Omega}\right)$ is a transverse Kähler foliation on $M$. By modifying the argument of Proposition 4.4 in [8], we obtain

$$
H^{1}\left(\sharp_{\Omega}\right)=H_{\mathrm{B}}^{n, 0}(M) \oplus H_{\mathrm{B}}^{n-1,1}(M), \quad H^{2}\left(\sharp_{\Omega}\right)=H_{\mathrm{B}}^{n, 1}(M) \oplus H_{\mathrm{B}}^{n-1,2}(M) .
$$

The maps $p^{1}$ and $p^{2}$ are injective by Proposition 2.3. Applying Theorem 4.1 to the elliptic orbit $\mathcal{O}_{\mathrm{SL}}$ completes the proof.

\section{$\S 6.3$. Transverse Calabi-Yau structures}

Definition 6.3. A pair $(\Omega, \omega) \in \bigwedge_{\mathrm{B}}^{n} \otimes \mathbb{C} \oplus \bigwedge_{\mathrm{B}}^{2}$ is called a transverse Calabi-Yau structure on $(M, \mathcal{F})$ if $\Omega$ is a transverse $\mathrm{SL}_{n}(\mathbb{C})$ structure and $\omega$ is a transverse symplectic structure on $(M, \mathcal{F})$ such that

$\Omega \wedge \omega=0, \quad \Omega \wedge \bar{\Omega}=c_{n} \omega^{n} \neq 0, \quad \omega\left(\cdot, J_{\Omega} \cdot\right)$ is positive definite on $Q$, where $c_{n}=\frac{1}{n !}(-1)^{n(n-1) / 2}(2 / \sqrt{-1})^{n}$. 
We denote by $\widetilde{\mathfrak{M}}_{\mathrm{CY}}(M, \mathcal{F})$ the space of transverse Calabi-Yau structures on $(M, \mathcal{F})$. Any element $\Phi=(\Omega, \omega)$ of $\widetilde{\mathfrak{M}}_{\mathrm{CY}}(M, \mathcal{F})$ is a transverse calibration associated with the elliptic orbit $\mathcal{O}_{\mathrm{CY}}$.

Proposition 6.2. The maps $p^{1}$ and $p^{2}$ are injective for any $\Phi \in \widetilde{\mathfrak{M}}_{\mathrm{CY}}(M, \mathcal{F})$.

Proof. Given $\Phi \in \widetilde{\mathfrak{M}}_{\mathrm{CY}}(M, \mathcal{F})$, by adapting the computation of cohomology groups in [8, Theorem 4.8] to basic forms we obtain

$$
\begin{aligned}
& H^{1}\left(\sharp_{\Phi}\right)=H_{\mathrm{B}}^{n, 0}(M) \oplus H_{\mathrm{B}}^{n-1,1}(M) \oplus \mathbb{P}_{B, \mathbb{R}}^{1,1}, \\
& H^{2}\left(\sharp_{\Phi}\right)=H_{\mathrm{B}}^{n, 1}(M) \oplus H_{\mathrm{B}}^{n-1,2}(M) \oplus\left(H_{\mathrm{B}}^{2,1}(M) \oplus H_{\mathrm{B}}^{1,2}(M)\right)_{\mathbb{R}}
\end{aligned}
$$

where $\left(H_{\mathrm{B}}^{2,1}(M) \oplus H_{\mathrm{B}}^{1,2}(M)\right)_{\mathbb{R}}$ and $\mathbb{P}_{B, \mathbb{R}}^{1,1}$ denote the real part of $H_{\mathrm{B}}^{2,1}(M) \oplus H_{\mathrm{B}}^{1,2}(M)$ and the space of real harmonic and primitive basic $(1,1)$-forms, respectively. Hence the maps

$$
\begin{aligned}
& p^{1}: H^{1}\left(\sharp_{\Phi}\right) \rightarrow H_{\mathrm{B}}^{n}(M, \mathbb{C}) \oplus H_{\mathrm{B}}^{2}(M), \\
& p^{2}: H^{2}\left(\sharp_{\Phi}\right) \rightarrow H_{\mathrm{B}}^{n+1}(M, \mathbb{C}) \oplus H_{\mathrm{B}}^{3}(M)
\end{aligned}
$$

are injective from Proposition 2.3 and the Lefschetz decomposition of basic differential forms (cf. [5, Section 3.4.7]).

We obtain the following results:

Theorem 6.2. Any element of $\widetilde{\mathfrak{M}}_{\mathrm{CY}}(M, \mathcal{F})$ is unobstructed.

Proof. This follows immediately from Theorem 4.1 and Proposition 6.2

Theorem 6.3. Let $\left\{\left(\Omega_{t}, \omega_{t}\right)\right\}_{t \in[0,1]}$ be a smooth family of transverse Calabi-Yau structures on $(M, \mathcal{F})$ with the same basic cohomology class $\left(\left[\Omega_{t}\right],\left[\omega_{t}\right]\right)=\left(\left[\Omega_{0}\right],\left[\omega_{0}\right]\right)$ $\in H_{\mathrm{B}}^{n}(M, \mathbb{C}) \oplus H_{\mathrm{B}}^{2}(M)$ for any $t \in[0,1]$. Then there exists a smooth family $\left\{f_{t}\right\}_{t \in[0,1]}$ in $\operatorname{Diff}_{0}(M, \mathcal{F})$ such that $f_{0}=\operatorname{id}_{M}$ and $\left(f_{t}^{*} \Omega_{t}, f_{t}^{*} \omega_{t}\right)=\left(\Omega_{0}, \omega_{0}\right)$.

Proof. Apply Theorem 5.1 and Proposition 6.2.

Remark. We can also obtain Moser's stability theorems and unobstructed deformations of transverse hyperkähler, $G_{2}$ and $\operatorname{Spin}(7)$ structures by applying Theorems 4.1 and 5.1 to these structures (cf. Sections 5, 6 and 7 in 8 ).

\section{§6.4. Linear foliations on tori}

Let $T^{2 n+1}$ be the real torus $\mathbb{R}^{2 n+1} / \mathbb{Z}^{2 n+1}$ of dimension $2 n+1$. We take a local coordinate $\left(x_{1}, \ldots, x_{n}, y_{1}, \ldots, y_{n}, t\right)$ on $T^{2 n+1}$. Then a foliation $\mathcal{F}_{(\lambda, \mu)}$ is induced by the vector field

$$
\xi=\sum_{i=1}^{n} \lambda_{i} \frac{\partial}{\partial x_{i}}+\mu_{i} \frac{\partial}{\partial y_{i}}-\frac{\partial}{\partial t}
$$


for $(\lambda, \mu)=\left(\lambda_{1}, \ldots, \lambda_{n}, \mu_{1}, \ldots, \mu_{n}\right) \in \mathbb{R}^{2 n}$. The foliation $\mathcal{F}_{(\lambda, \mu)}$ is called a linear foliation on $T^{2 n+1}$. Note that $\mathcal{F}_{(\lambda, \mu)}$ is a Riemannian foliation with respect to the standard flat metric on $T^{2 n+1}$. We define $z_{i}$ to be the complex function

$$
z_{i}=x_{i}+\lambda_{i} t+\sqrt{-1}\left(y_{i}+\mu_{i} t\right)
$$

for $i=1, \ldots, n$. Then $\left(z_{1}, \ldots, z_{n}\right)$ becomes a transverse coordinate on $\left(T^{2 n+1}, \mathcal{F}_{(\lambda, \mu)}\right)$. Now we define a pair $(\Omega, \omega)$ of forms as

$$
\Omega=d z_{1} \wedge \cdots \wedge d z_{n}, \quad \omega=\frac{\sqrt{-1}}{2} \sum_{i=1}^{n} d z_{i} \wedge d \bar{z}_{i} .
$$

Then it is easy to check that $(\Omega, \omega)$ is a transverse Calabi-Yau structure on $\left(T^{2 n+1}, \mathcal{F}_{(\lambda, \mu)}\right)$. In particular, the $n$-form $\Omega$ is a transverse $\mathrm{SL}_{n}(\mathbb{C})$ structure so that $\left(\mathcal{F}_{(\lambda, \mu)}, J_{\Omega}\right)$ is a transverse Kähler foliation. Hence, it follows from Proposition 6.1 that $\Omega \in \widetilde{\mathfrak{M}}_{\mathrm{SL}}\left(T^{2 n+1}, \mathcal{F}_{(\lambda, \mu)}\right)$ is unobstructed and $H^{1}\left(\sharp_{\Omega}\right)$ equals $H_{\mathrm{B}}^{n, 0}\left(T^{2 n+1}\right) \oplus$ $H_{\mathrm{B}}^{n-1,1}\left(T^{2 n+1}\right)$. The vector space $H_{\mathrm{B}}^{p, q}\left(T^{2 n+1}\right)$ is generated by the classes of the wedge products $d z_{i_{1}} \wedge \cdots \wedge d z_{i_{p}} \wedge d \bar{z}_{j_{1}} \wedge \cdots \wedge \bar{z}_{j_{q}}$, and thus we obtain

$$
\operatorname{dim}_{\mathbb{C}} H_{\mathrm{B}}^{p, q}\left(T^{2 n+1}\right)=\left(\begin{array}{l}
n \\
p
\end{array}\right)\left(\begin{array}{l}
n \\
q
\end{array}\right) .
$$

The first cohomology group $H^{1}\left(\sharp_{\Omega}\right)$ can be regarded as a parameter space of deformations of the transverse $\mathrm{SL}_{n}(\mathbb{C})$ structure $\Omega$, and has the dimension

$$
\operatorname{dim}_{\mathbb{C}} H^{1}\left(\sharp_{\Omega}\right)=\operatorname{dim}_{\mathbb{C}}\left(H_{\mathrm{B}}^{n, 0}\left(T^{2 n+1}\right) \oplus H_{\mathrm{B}}^{n-1,1}\left(T^{2 n+1}\right)\right)=1+n^{2} .
$$

Next, we consider the deformations of transverse Calabi-Yau structures on $\left(T^{2 n+1}, \mathcal{F}_{(\lambda, \mu)}\right)$. By Theorem 6.2, any element $\Phi \in \widetilde{\mathfrak{M}}_{\mathrm{CY}}\left(T^{2 n+1}, \mathcal{F}_{(\lambda, \mu)}\right)$ is unobstructed and $H^{1}\left(\sharp_{\Phi}\right)$ is equal to $H_{\mathrm{B}}^{n, 0}\left(T^{2 n+1}\right) \oplus H_{\mathrm{B}}^{n-1,1}\left(T^{2 n+1}\right) \oplus \mathbb{P}_{B, \mathbb{R}}^{1,1}$. It follows that

$$
\operatorname{dim}_{\mathbb{R}} \mathbb{P}_{B, \mathbb{R}}^{1,1}=\operatorname{dim}_{\mathbb{C}} \mathbb{P}_{B}^{1,1}=n^{2}-1
$$

from the basic Lefschetz decomposition $H_{\mathrm{B}}^{1,1}\left(T^{2 n+1}\right)=\mathbb{P}_{\mathrm{B}}^{1,1}+\mathbb{C} \omega$. Hence, any element $\Phi \in \widetilde{\mathfrak{M}}_{\mathrm{CY}}\left(T^{2 n+1}, \mathcal{F}_{(\lambda, \mu)}\right)$ essentially has a deformation space parameterized by $H^{1}\left(\sharp_{\Phi}\right)$ whose dimension is

$$
\operatorname{dim}_{\mathbb{R}} H^{1}\left(\sharp_{\Phi}\right)=2\left(1+n^{2}\right)+n^{2}-1=3 n^{2}+1
$$

\section{Acknowledgements}

The author is very grateful to Professor R. Goto for his advice. He also thanks the referee for his careful reading of the paper and useful comments. 
This research was supported by GCOE 'Fostering top leaders in mathematics', Kyoto University and by Grant-in-Aid for Young Scientists (B) $\sharp 21740051$ from JSPS.

\section{References}

[1] R. Barre and A. El Kacimi-Alaoui, Foliations, in Handbook of differential geometry, Vol. II, Elsevier/North-Holland, Amsterdam, 2006, 35-77. Zbl 1140.57301 MR 2194668

[2] C. P. Boyer and K. Galicki, Sasakian geometry, Oxford Math. Monogr., Oxford Univ. Press, Oxford, 2008. Zbl 1155.53002 MR 2382957

[3] M. Crainic and R. L. Fernandes, Rigidity and flexibility in Poisson geometry, in Travaux mathématiques, Fasc. XVI, Univ. Luxembourg, 2005, 53-68. Zbl 1097.53053| MR 2223151

[4] T. Duchamp and M. Kalka, Deformation theory for holomorphic foliations, J. Differential Geom. 14 (1979), 317-337. Zbl 0451.57015 MR 0594704

[5] A. El Kacimi-Alaoui, Opérateurs transversalement elliptiques sur un feuilletage riemannien et applications, Compos. Math. 73 (1990), 57-106. Zbl 0697.57014 MR 1042454

[6] J. Girbau, A. Haefliger and D. Sundararaman, On deformations of transversely holomorphic foliations, J. Reine Angew. Math. 345 (1983), 122-147. Zbl 0538.32015 MR 0717890

[7] X. Gomez-Mont, Transversal holomorphic structures, J. Differential Geom. 15 (1980), 161186. Zbl 0492.57009 MR 0614365

[8] R. Goto, Moduli spaces of topological calibrations, Calabi-Yau, hyperkähler, $G_{2}$ and $\operatorname{Spin}(7)$ structures, Int. J. Math. 15 (2004), 211-257. Zbl 1046.58002 MR 2060789

[9] R. Harvey and H. B. Lawson, Calibrated geometries, Acta Math. 148 (1982), 47-157. Zbl 0584.53021 MR 0666108

[10] K. Kodaira and D. C. Spencer, On deformation of complex analytic structures I and II, Ann. of Math. 67 (1958), 328-466. MR 0112154

[11] M. Kuranishi, Deformations of compact complex manifolds, Sém. Math. Sup. 39, Presses Univ. de Montréal, 1971. MR 0355111

[12] R. C. McLean, Deformation of calibrated submanifolds, Comm. Anal. Geom. 6 (1998), 705-749. Zbl 0929.53027 MR 1664890

[13] P. Molino, Riemannian foliations, Birkhäuser, 1988. Zbl 0633.53001 MR 0932463

[14] P. Tondeur, Geometry of foliations, Monogr. Math. 90, Birkhäuser, 1997. Zbl 0905.53002 MR 1456994 